\title{
Aprendizaje basado en proyectos en contexto: estrategia para desarrollar el razonamiento estadístico
}

\author{
Smith Barrios Sandoval* \\ Ana Cecilia Medina Mariño**
}

Artículo de reflexión

Fecha de Recepción: 15 diciembre 2017.

Fecha de Aprobación: 30 abril 2018.

\section{Resumen}

Este artículo da a conocer el avance de una investigación en el aula con enfoque cualitativo, que tiene como propósito desarrollar el razonamiento estadístico en niños de grado tercero de Educación Básica Primaria, mediante la estrategia de aprendizaje basado en proyectos (ABPr), a partir de un problema del contexto. En la revisión teórica y construcción de los antecedentes se reconoce la necesidad de los datos como base de la investigación estadística para comprender situaciones de la vida real. Se presenta a manera de reflexión, los resultados de la fase diagnóstica orientada a identificar los niveles iniciales de razonamiento estadístico que muestran los niños, con relación a la lectura e interpretación de tablas y gráficas estadísticas. La información analizada proviene de la aplicación de una prueba basada en una situación del contexto, un cuestionario utilizado por Ríos (2014) y una entrevista a docentes de la institución. Se encontró que los niños se ubican en los niveles de razonamiento estadístico idiosincrático y verbal según Garfield, Del Mas y Chance (1999). También se infieren algunos factores que inciden en este hallazgo, y al reconocerlos se corrobora que la estrategia planteada puede contribuir a mejorar el pensamiento aleatorio.

Palabras clave: Razonamiento estadístico, aprendizaje basado en proyectos, niveles de razonamiento, metodología en Proyectos
*Institución Educativa Marco Antonio Quijano Rico-BoyacáColombia smith.barrios03@gmail.com

** Docente Universidad Pedagógica y Tecnológica de Colombia-Boyacá-Colombia ana.medina@uptc.edu.co

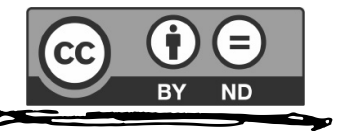




\section{Introducción}

La enseñanza y aprendizaje de la estadística desde los primeros años escolares se ha convertido en una necesidad y tendencia internacional, con el fin de generar y desarrollar gradualmente la cultura y el razonamiento estadístico, necesario para que los futuros ciudadanos estén en capacidad de analizar críticamente e interpretar información estadística, proveniente del comportamiento de fenómenos de contextos sociales, políticos, económicos, científicos y culturales del mundo que los rodea (Batanero, 2013; Tauber, 2010; Inzunsa, 2015). Esto les ayudará a tomar decisiones acertadas que puedan aportar favorablemente a la solución de problemas de los distintos contextos en que se encuentren involucrados. Para tratar de contribuir al alcance de estas metas educativas, y a partir de la reflexión sobre la práctica pedagógica y el currículo escolar implementado, se dio inicio a la investigación analizando los aportes de diferentes autores con el ánimo de revisar y actualizar contenidos curriculares y estrategias didácticas, que orientan la aplicación de actividades de investigación en forma asertiva y proporcionan ideas prácticas que sirven de apoyo al docente en el aula.

Autores como Batanero, Díaz, Contreras \& Roa (2013) destacan la importancia de la estadística inseparable de sus aplicaciones y su justificación es la utilidad en la resolución de problemas externos a la propia estadística. E1 aporte de este trabajo a la investigación está referido a los conceptos de cultura estadística, el razonamiento estadístico y la metodología en proyectos, ya que al trabajar con proyectos se sitúa a los alumnos en la posición de tener que pensar en preguntas como las siguientes: ¿Cuál es mi problema? ¿Necesito datos? ¿Cuáles? ¿Cómo puedo obtenerlos? ¿Qué significa este resultado en la práctica? (Graham, 1987).

Por otra parte, Batanero y Díaz (2004), recomiendan introducir en las clases de razonamiento estadístico el ABPr, algunos planteados por el profesor otros por los estudiantes. No se trata de introducir conceptos y técnicas descontextualizados, se trata de presentar las fases de la investigación estadística planteamiento del problema, decisión sobre los datos a recoger, recogida y análisis de datos y obtención de conclusiones sobre el problema planteado. Además, expresan que un buen proyecto se caracteriza por que sus actividades se desarrollan dentro de un contexto real.

De la misma manera, Holmes (1997) expresa si los estudiantes trabajan la Estadística por medio de proyectos se consiguen varios puntos positivos: los proyectos permiten contextualizar la estadística y hacerla más relevante, los datos surgen de un problema, son datos con significado y tienen que ser interpretados; asimismo los proyectos refuerzan el interés, sobre todo si es el alumno es el que elige el tema, pues él quiere resolver el problema y al no ser impuesto por el profesor se aprende mejor, ya que los datos son reales y, se 
introducen ideas que no aparecen con los "datos inventados por el profesor" como: precisión, variabilidad, fiabilidad, posibilidad de medición y sesgo, entonces se muestra que la estadística no se reduce a contenidos matemáticos.

Entre tanto, Wild y Pfannkuch (1999, citado por Tapiero \& Polanco, 2014) conciben el razonamiento estadístico basándose en cuatro dimensiones, desde el ciclo de investigación: problema, el plan, los datos, el análisis y las conclusiones; los modos fundamentales del razonamiento estadístico; el ciclo de interrogación y una serie de actitudes. Comúnmente las aulas se caracterizan por el uso del conocimiento técnico, memorización de conceptos, fórmulas, lectura de gráficos, búsqueda de una única solución, dificultad para la aplicación de procesos y formulación de problemas. Pero, no se puede obviar que la estadística es una herramienta indispensable que se aplica en la vida cotidiana, en el contexto de todo ser humano que pretende desarrollar los conceptos de cultura estadística, razonamiento estadístico y proponer el trabajo por proyectos como estrategia metodológica para desarrollar el sentido estadístico. Para esta investigación los autores son importantes por cuanto la temática está en el contexto del trabajo de grado.

Connor, Davies \& Payne (2002) indican que cada vez es más frecuente la realización de estos proyectos, debido a que en el currículo de matemáticas se contempla la realización de proyectos. Estos varían desde problemas sencillos de representación de datos, hasta la comprobación de hipótesis o el uso de la simulación. Las nuevas propuestas curriculares son una oportunidad para introducir gradualmente el razonamiento estadístico, a partir de la estrategia metodológica por proyectos deinterés del niño, en lugar de introducir conceptos descontextualizados, que no tienen aplicabilidad en la vida real.

De igual manera, en el estudio realizado por Godino, Arteaga, Estepa \& Rivas (2013) y Jimenez, Limas y Alarcón (2016) se destaca la enseñanza de la estadística a partir de situaciones en contexto producto del análisis de datos, empleo de elementos lingüísticos, proposiciones y argumentos e implementación de momentos de exploración, formulación, comunicación, validación, ejercitación y evaluación.

El trabajo de Tapiero \& Polanco (2014) promueve el desarrollo del razonamiento estadístico a través del ABPr, utilizando el ciclo de investigación: problema, plan, datos, análisis y conclusiones y los modos fundamentales del razonamiento $y$ las actitudes, propuesto por Wild y Pfannkuch (1999). El aporte de este trabajo a la investigación está en la propuesta del modelo para establecer las categorías del desarrollo del razonamiento estadístico en cada uno de los estudiantes: razonamiento idiosincrático, verbal, transición, procesos y procesos integrados, las cuales se describen sintéticamente en el desarrollo metodológico (Garfield, Del Mas \& Chance, 1999, citado por Tapiero \& Polanco, 2014).
Wild y Pfannkuch (1999, citado por Tapiero \& Polanco, 2014) conciben el razonamiento estadístico basándose en cuatro dimensiones, desde el ciclo de investigación: problema, el plan, los datos, el análisis y las conclusiones 


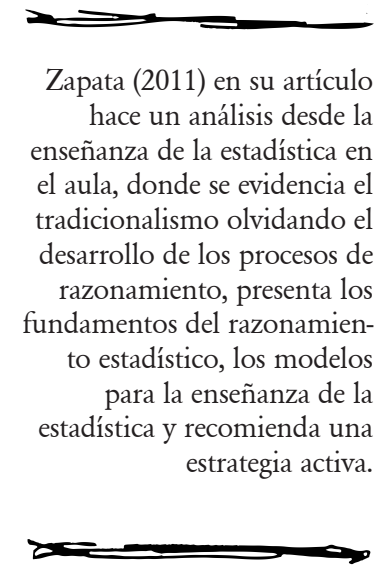

Por su parte Zapata (2011) en su artículo hace un análisis desde la enseñanza de la estadística en el aula, donde se evidencia el tradicionalismo olvidando el desarrollo de los procesos de razonamiento, presenta los fundamentos del razonamiento estadístico, los modelos para la enseñanza de la estadística y recomienda una estrategia activa. El aporte de este estudio al proyecto de investigación tiene que ver con el constructo del razonamiento estadístico desde el diseño de la pregunta, diseño de experimentos, recogida de datos, análisis, procedimientos, y conclusiones, el optar por una estrategia activa de aprendizaje por proyectos, y los elementos fundamentales del razonamiento estadístico planteados por Wild \& Pfannkuch (1999, citado por Tapiero \& Polanco, 2014), quien toma en consideración: la variación, la transnumeración, el razonamiento a partir de modelos, la síntesis del problema en contextos particulares y la comprensión estadística. Algunos atributos personales, como la imaginación, la lógica, el escepticismo y la curiosidad, también son importantes en el razonamiento estadístico.

De otro lado, Gil \& Rocha (2010) presentan una reflexión sobre la relevancia y el aprendizaje de la estadística, producto de la utilización de los datos en la vida cotidiana, medios de comunicación, investigaciones, vida laboral, como educadores estadísticos capaces de contribuir en la construcción de las ideas estadísticas y su aplicación en el contexto aporta a la investigación desde las fases para el desarrollo de un proyecto: propuesta, planificación, elaboración y evaluación.
Córdoba (2012) reflexiona acerca de la enseñanza de la estadística expresando la importancia de su aplicabilidad en el contexto real del estudiante para evitar la transmisión de conceptos y como desde la propuesta aprendizaje basado en el trabajo por proyectos se construye el conocimiento de una forma más significativa, sin descuidar lo conceptual y procedimental y desde esta mirada aporta al proyecto de investigación.

Ahora bien, aunque la estadística se enseña hoy día en todos los niveles de la institución educativa donde se desarrolla el presente trabajo investigativo, en el quehacer pedagógico se observa que la mayoría de estudiantes al finalizar un año escolar, terminan sin comprender los conceptos o ideas estadísticas y difícilmente relacionan los conceptos con los procedimientos, para solucionar un problema desde la cotidianidad; por lo tanto, tienen dificultades para plantear preguntas o problemas, formular hipótesis $\mathrm{y}$ relacionar saberes previos con los nuevos conocimientos. Al respecto Batanero et al. (2013), aluden que desde las prácticas pedagógicas el docente promueve el aprendizaje de la estadística de manera memorística, basada en fórmulas y descontextualizada de los intereses del niño y situaciones reales, donde los contenidos que más se estudian en estos cursos son los referidos a la elaboración e interpretación de tablas y gráficos.

Otro factor que puede incidir en la problemática es el nivel educativo de los padres de familia, pues en su 
mayoría no terminaron la primaria, los núcleos familiares numerosos, la cultura del trabajo, donde lo más importante es enseñar a los niños las labores del campo y del hogar, según datos obtenidos y registrados en el momento de la matrícula. Así, un factor determinante en el desempeño escolar, puede estar relacionado con las condiciones de pobreza en el entorno familiar; por ejemplo, en la mayoría de los casos las viviendas de los niños tienen un solo cuarto y este sitio se convierte en el comedor, sala de estudio, habitación entre otros; además sus viviendas no cuentan con alcantarillado, los pisos en su mayoría son de tierra y no poseen herramientas tecnológicas.

De igual forma, se observa que la problemática se presenta desde la planeación curricular, pues la estadística ha sido incorporada en el currículo con énfasis en contenidos, en forma descontextualizada, y generalmente se programa para el final del curso, razón por la cual usualmente no se alcanza a estudiar. El pensamiento aleatorio no se considera transversal con los demás tipos de pensamiento matemático, ni con las diferentes áreas del conocimiento. Al respecto, Holmes (1997) sugiere una oportunidad a partir de la estrategia ABPr estadísticos del contexto, para utilizar un enfoque interdisciplinario, resolver problemas cotidianos, aumentar la motivación del estudiante, crear datos a partir de situaciones reales, contextualizar la estadística y hacerla significativa. Como lo menciona Zapata (2011), el ciudadano común enfrenta el desafío permanente de leer e interpretar datos estadísticos que surgen de diferentes fuentes. Infortunadamente, nuestros ciudadanos cuentan con una alfabetización estadística insuficiente para enfrentar con éxito estos retos que la cultura le demanda.

En tal sentido, y al efectuarse el análisis de los resultados de las Pruebas Saber de tercero de primaria (2015) de la Institución Educativa, se evidenció que el 54\% de los estudiantes de este grado no están clasificando ni ordenando datos, el 54\% de los estudiantes no identifican atributos de objetos y eventos que son susceptibles de ser medidos, el 46\% de los estudiantes no representan un conjunto de datos a partir de un diagrama de barras, ni interpretan lo que un diagrama de barras determinado representa, el $58 \%$ de los estudiantes no resuelven problemas a partir del análisis de datos recolectados. Al contrastar con la matriz de referencia basada en los Estándares Básicos de Competencias (MEN, 2006), los estudiantes presentaron dificultades en la competencia razonamiento estadístico para describir tendencias que se muestran en un conjunto a partir de los datos que lo describen y establecer conjeturas acerca de la posibilidad de ocurrencia de eventos.

Sumado a lo anterior, la insatisfacción de los estudiantes, su inquietud, la falta de atención, concentración y desfavorables resultados como respuesta al paradigma de la pedagogía tradicional, memorística y repetitiva; cuando ellos han descubierto que son capaces de hacer algo más y diferente
El pensamiento aleatorio no se considera transversal con los demás tipos de pensamiento matemático, ni con las diferentes áreas del conocimiento. 


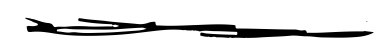

La investigación está enmarcada en el paradigma social-critico como respuesta al tradicionalismo en la educación: memorística, repetitiva, técnica, basada en conceptos descontextualizados y su poca influencia en la transformación social. a replicar la información dada por el docente, los libros o textos, transcribir del tablero al cuaderno.

Fue así que tomando como referencia la importancia del razonamiento estadístico se planteó la siguiente pregunta de investigación ¿Cómo el aprendizaje basado en proyectos del contexto contribuye a desarrollar el razonamiento estadístico en los estudiantes de grado tercero de una Institución Educativa del municipio de Sogamoso, Boyacá? Para lo cual se trazan propósitos como el desarrollo del razonamiento estadístico, mediado por logros como la realización de un diagnóstico sobre la caracterización de los estudiantes de grado tercero de la institución, teniendo en cuenta el nivel inicial de razonamiento estadístico; para posteriormente diseñar y aplicar la propuesta didáctica utilizando la estrategia ABPr del contexto, finalizando con la sistematización y evaluación de la estrategia.

\section{Desarrollo metodológico}

El trabajo se desarrolla mediante un enfoque cualitativo, lo cual implica que las actividades se encuentran categorizadas dentro de procesos de observación e indagación, para identificar las problemáticas relacionadas con el razonamiento estadístico presente en los estudiantes y con ello, realizar un cambio en las prácticas tradicionales a partir de la implementación del aprendizaje basado en proyectos.

Se retoma el enfoque cualitativo propuesto por Sandoval-Casilimas
(2002), como opción metodológica para apoyar la investigación socialcritica desde las formas de entender y conocer la realidad a partir de la lógica y sentir de la población objeto de estudio, hasta las formas de construir el conocimiento. El investigador entra a la realidad del objeto de análisis para comprender la lógica, emplea la subjetividad e intersubjetividad para conocer las realidades. Entre tanto, para la teoría socio-critica la construcción del conocimiento se da a partir de la indagación y está se estructura a partir de hallazgos que se van realizando durante el transcurso de la investigación, y la validación de conclusiones se hace a través del diálogo, la interacción y la vivencia nacidos de los procesos de observación, reflexión, construcción de sentido compartido y sistematización.

La investigación está enmarcada en el paradigma social-critico como respuesta al tradicionalismo en la educación: memorística, repetitiva, técnica, basada en conceptos descontextualizados y su poca influencia en la transformación social. Además, brinda los aportes para el cambio social desde el interior de la comunidad educativa y su finalidad es dar respuestas a problemas, partiendo de la acción-reflexión de los integrantes de la comunidad. Se considera como una unidad entre lo teórico y lo práctico, que pretende descubrir la verdad mediante la construcción de argumentos contrarios entre sí. Nace de una crítica a la racionalidad instrumental y técnica, plantea la 
necesidad de una racionalidad subjetiva que incluya juicios, valores, intereses de la sociedad, así como un compromiso para transformación desde su interior (Alvarado \& García, 2008).

La finalidad de esta investigación es mejorar las prácticas docentes, modificar un problema desde el contexto escolar que repercuta en el mejoramiento de la calidad educativa. Por esta razón, se considera la Investigación -Acción (IA) como la más indicada para resolver problemas en el aula. Así, el desarrollo metodológico del estudio se realiza atendiendo a las características y etapas de la I-A propuestas por Lewin (1944):

Lewin bosqueja un proceso disciplinado de investigación- acción que se sitúa en paralelo con la aplicación del Método científico en otras disciplinas. Su modelo especifica una espiral de actividades en esta secuencia:

\section{Aclaración y diagnóstico de una} situación problemática en la práctica. 2. Formulación de estrategias de acción para resolver el problema. 3. Implantación y evaluación de las estrategias de acción y 4. Aclaración y diagnóstico posteriores de las situación problemática (y así sucesivamente en la siguiente espiral de reflexión y acción) (Elliot, 1994, p.97).

En el Reino Unido Elliot (1994) ha promovido la I-A en la escuela caracterizándola de la siguiente forma: la I-A se relaciona con los problemas prácticos cotidianos experimentados por los profesores, en vez de con los "problemas teóricos" definidos por los investigadores puros en el entorno de una disciplina; el propósito de la I-A consiste en profundizar la comprensión del profesor (diagnóstico) de su problema; adopta una postura teórica para conseguir una comprensión más profunda del problema práctico en cuestión; interpreta lo que ocurre desde el punto de vista de quienes actúan e interactúan en la situación problema. Todo con el fin de mejorar las prácticas educativas dentro y fuera del aula sometidas a la observación, reflexión y al cambio.

Es en la primera etapa de la I-A que se realiza el diagnóstico sobre los niveles iniciales de razonamiento estadístico que manifiestan los estudiantes de grado tercero, a la hora de leer e interpretar datos en tablas y gráficas estadísticas, y cuyos resultados se muestran a continuación. Ahora, se emprende la segunda etapa de formulación de estrategias de acción adoptando la teoría de Aprendizaje de la Estadística Basada en Proyectos, teniendo en cuenta las fases de una investigación estadística: identificación de un problema, planteamiento de preguntas, decisión sobre los datos a recoger, organización, interpretación y análisis de datos y obtención de conclusiones sobre el problema planteado (Batanero \& Díaz, 2011).

El contexto donde se desarrolla el proyecto deinvestigación es la Institución Educativa Marco Antonio Quijano Rico, sede el Hatillo, del municipio de Sogamoso (Colombia). Plantel oficial con un enfoque pedagógico de Formación Basada en Competencias, un modelo constructivista que favorece 


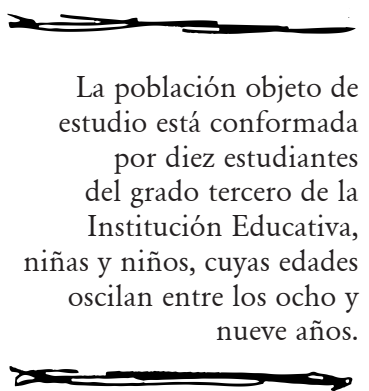

el desarrollo de la estadística a partir del aprendizaje por proyectos, que como estrategia de enseñanza constituye un modelo de instrucción auténtico en el que los estudiantes planean, implementan y evalúan proyectos que tienen aplicación en el mundo real más allá del aula de clase (Blank, 1997; Dickinson et al, 1998; Harwell, 1997, citados en NorthWest Regional Educational Laboratory, s.f.).

La población objeto de estudio está conformada por diez estudiantes del grado tercero de la Institución Educativa, niñas y niños, cuyas edades oscilan entre los ocho y nueve años. Se caracterizan por ser hijos de padres campesinos dedicados a las labores del campo, pertenecientes a un nivel socio económico bajo, por lo que habitan en condiciones muy precarias. En el aula muestran dificultades para relacionar los saberes previos con la construcción de nuevos conocimientos en forma significativa.

Para la recopilación y análisis de la información se diseñaron y aplicaron los siguientes instrumentos, tras la obtención de un consentimiento informado: una prueba basada en una situación adaptada de los Derechos Básicos de Aprendizaje (MEN, 2016), un cuestionario que consta de ocho ítems de selección múltiple con única respuesta, tomado del trabajo de Ríos (2014, p.104,105): “Estadística para pequeños estadísticos - construcción de unidades didácticas y material de apoyo", entrevistas a algunos docentes de primaria y una rúbrica de evaluación de la competencia de razonamiento, según los niveles de razonamiento estadístico tomando descriptores propuestos por Garfield et al.(1999), citado por Tapiero \& Polanco (2014):

Nivel 1. Razonamiento Idiosincrático: es aquel que muestra el conocimiento del estudiante con respecto al lenguaje (palabras y símbolos), aunque no lo usan ni entienden de forma correcta. Presentan dificultad para organizar, clasificar e interpretar datos. Nivel 2. Razonamiento Verbal: cuando los estudiantes tienen un entendimiento verbal del objeto matemático, pero no lo aplican de manera correcta. Nivel 3. Razonamiento de Transición: los estudiantes son capaces de identificar una o dos características del objeto matemático pero no integra completamente esas características. Nivel 4. Razonamiento de procesos: los estudiantes identifican todas las características de los datos pero no hacen una integración total de ellas. Nivel 5. Razonamiento de procesos integrados: los estudiantes tienen un entendimiento total del objeto, explican con sus propias palabras, describen los resultados obtenidos (p.283).

\section{Resultados y discusión}

A continuación se presentan los resultados y el análisis de la información proveniente de la aplicación de los instrumentos mencionados anteriormente:

En relación con la prueba cuyo objetivo era determinar el nivel de razonamiento estadístico que muestran los niños, según el modelo de Garfield et al. (1999) citado por Tapiero \& Polanco (2014), y en la que se plantea la siguiente situación: 
El director de la escuela bizo una encuesta acerca del medio que utilizan los niños para acceder a la escuela y el tiempo que gastan. Luego de la encuesta, solicita a los alumnos su colaboración para que le propongan títulos adecuados a la tabla 1 y la figura 1 $y$ que además escriban un informe corto con el análisis de los resultados.

Tabla 1. Información suministrada en el ítem

\begin{tabular}{|l|l|}
\hline Caminando & 10 \\
\hline Bicicleta & 14 \\
\hline Bus & 25 \\
\hline Taxi & 5 \\
\hline Carro Particular & 12 \\
\hline Total & 66 \\
\hline
\end{tabular}

Figura 1. Información ítem 1.

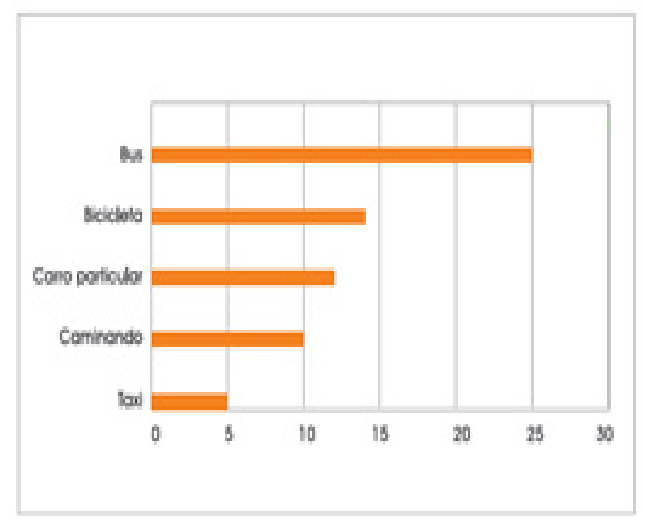

Fuente: MEN (2016)

Luego, el trabajo de los niños consistía en identificar la información contenida en cada representación y proponer títulos coherentes con una posible pregunta de estudio. En este caso, de los diez estudiantes, ninguno pudo leer ni interpretar la tabla y la gráfica. No escribieron el título y no relacionan ningún informe sobre el análisis. Al analizar los resultados se puede inferir que los niños desconocen totalmente este tipo de representación de información. Además, manifiestan dificultad para comprender la situación, y parece que todavía tienen inconvenientes en lectura y escritura de textos.

Continuando con la indagación se aplica un cuestionario para verificar en qué nivel de razonamiento estadístico se encuentra cada niño. El cuestionario consta de ocho ítems de selección múltiple con única respuesta, tomados del trabajo de Ríos (2014). Los niños deben leer detenidamente la pregunta, analizarla y seguidamente emitir la respuesta que crea acertada. En la figura 2 se consolida los resultados obtenidos en el cuestionario descrito anteriormente.

Figura 2. Número de estudiantes que responden acertadamente los ítems del cuestionario.

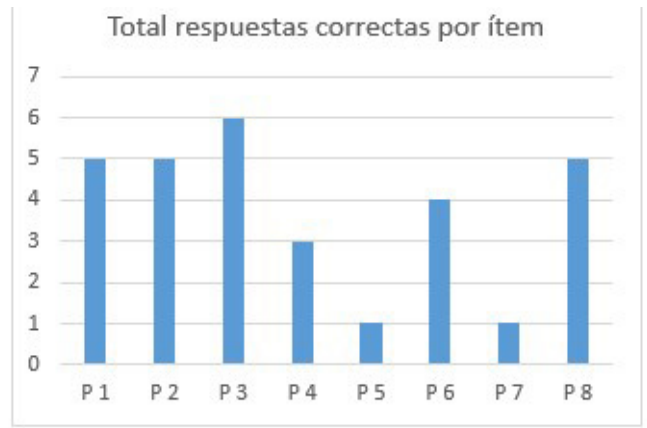

Fuente: Las autoras.

Las preguntas uno y dos las debían contestar de acuerdo con una situación en contexto que describe un jardín con animales que allí habitan: mariposas, caracoles, mariquitas, gusanos, abejas y arañas.

Al confrontar las respuestas de los niños con respecto a la pregunta uno, que consistía en interpretar 
la información de la tabla y elegir de cuatro alternativas la respuesta correcta, se observa que cinco de los diez niños contestaron acertadamente. Sin embargo, cabe anotar que los niños que no relacionaron el número de cuadros con la cantidad que representaba cada cuadro sombreado (dos animales) o los que no tienen una adecuada comprensión lectora, no acertaron con la respuesta correcta.

El cuanto a la pregunta dos, que exigía mayor observación e interpretación de proposiciones lingüísticas que expresan relación de orden, solo cuatro estudiantes acertaron en la respuesta, lo cual deja entrever que no solo la comprensión lectora es importante, sino también el uso e interpretación de proposiciones matemáticas como "había más...que..." , "había solo..." , expresiones que para los niños, es posible que no tengan suficiente dominio y por tal razón se equivocan al emitir la respuesta.

Las preguntas tres, cuatro y cinco se respondían con base en la información proporcionada por un grupo de estudiantes a los que su profesor les preguntó sobre cuál era el animal doméstico que más les gustaba y los estudiantes expresan en forma repetida su gusto nombrando sucesivamente animales domésticos como: gallina, conejo, perro, gato y cerdo.

En la pregunta número tres, los niños deben identificar entre cuatro tablas de conteo y frecuencia que se proporcionan, cuál es la tabla correcta que tiene la información enunciada por el profesor. En el cuarto ítem, deben hacer comparaciones sobre el animal de mayor o menor gusto con respeto a los demás, es decir comparar frecuencias absolutas, y en la cinco debían relacionar la frecuencia con el diagrama de barras que corresponde a la situación.

$\mathrm{Al}$ analizar las respuestas se evidencia que, a pesar de la sencillez de los temas, en la medida en que aumenta el grado de complejidad, va bajando el número de respuestas acertadas; en la pregunta tres, por ejemplo, solo cuatro niños de los diez responden acertadamente. Esto demuestra que los niños no comprenden la manera de obtener la frecuencia en una información dada, no hay que desconocer que las tablas de las respuestas proporcionan el conteo y la cantidad en números; luego surge la inquietud de si los niños presentan problemas de conteo. Entre tanto, las respuestas dadas a la pregunta cuatro reflejan que la mayoría de los niños no saben identificar la moda de un conjunto de datos, pues solo tres contestaron acertadamente.

Cabe hacer énfasis, nuevamente, en las dificultades manifestadas por los estudiantes en la comprensión lectora, pues, afirmaciones como le gusta más...que...; el animal que más les gusta después de..., son expresiones lingüísticas que no son comunes para los niños; por tal razón, se supone que se les dificulta su comprensión y por ende seleccionan erróneamente la respuesta. En la pregunta cinco donde solo un niño acertó la respuesta, se puede inferir que la mayoría de ellos no reconocen los gráficos estadísticos y no establecen 
las relaciones correspondientes entre las tablas de frecuencias y los gráficos presentados.

La pregunta seis utiliza como contexto una cafetería, allí venden jugos de frutas; el niño debe analizar la frecuencia y determinar cuál es el jugo que más les gusta a las personas que los visitan durante una semana, con base en un diagrama de barras. La pregunta es contestada acertadamente por cuatro de diez niños que desarrollan el cuestionario, se confirma lo mencionado en el numeral anterior, la mayoría de los niños desconocen como hallar la frecuencia de un suceso y no lo saben relacionar con las gráficas estadísticas existentes, para el caso un diagrama de barras. Esto explica porque los niños no contestaron a la pregunta uno de la primera prueba.

Las preguntas siete y ocho se contestaban conbase en la información proporcionada por el siguiente enunciado:

En una población se preguntó sobre el medio de transporte que más utilizaban sus habitantes y los resultados se presentaron mediante la figura 3.

Figura 3. Información para solucionar las preguntas siete y ocho. Fuente: Ríos (2014, p.106)

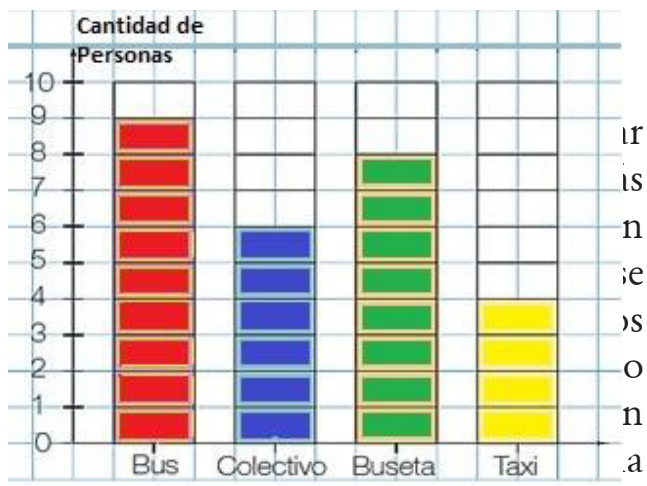

frecuencia de los datos de un suceso y que la comprensión lectora puede hasta cierto punto, influir negativamente en la manera como los niños asignan significado a la pregunta y a la representación gráfica.

Finalmente, en la pregunta ocho los niños debían asociar la altura de la barra con la cantidad de personas y relacionar estas cantidades. Fue contestada acertadamente por cinco estudiantes, es importante mencionar la inquietud planteada al inicio del análisis de la prueba, puede ser que los niños presenten dificultades con el conteo y su relación con la lectura de gráficas estadísticas.

Partiendo del análisis de las respuestas de cada ítem del cuestionario aplicado a los diez estudiantes y de la información arrojada en la primera prueba, a partir de la lectura de una situación del contexto, se puede llegar a las siguientes disertaciones:

Todo instrumento diagnóstico responde a unas intencionalidades que, para el caso de la presente investigación, estuvieron en relación con la identificación de los niveles iniciales de razonamiento estadístico en que se encuentran los niños de grado tercero, según el modelo de Garfield et al. (1999), citado por Tapiero y Polanco (2014).

Se identificó que los mejores desempeños se logran en la relación existente entre la cantidad y la frecuencia, no se evidencia un nivel de comprensión básico del lenguaje de la Estadística, es decir, no reconocen artiendo del análisis de las respuestas de cada ítem del cuestionario aplicado a los diez estudiantes y de la información arrojada en la primera prueba 
representaciones gráficas, ni algunas medidas de tendencia central como la moda y tampoco manejan escalas de representación de datos.

Es importante resaltar que de las ocho preguntas del cuestionario dos fueron contestadas acertadamente por la mitad de los estudiantes, tres de las preguntas muestran una constante de cuatro acertadas y el resto equivocadas, una donde solo tres contestaron acertadamente y dos donde solo una pregunta fue contestada correctamente. Ello evidencia el desconocimiento de estrategias para la lectura e interpretación de textos en lenguaje habitual e información estadística en tablas y gráficas, por las dificultades reveladas al relacionar la cantidad con la frecuencia, así como la lectura e interpretación en general.

Se concluye que el 90\% de los estudiantes tienen dificultades para identificar la frecuencia en un diagrama de barras, el 70\% de los estudiantes no pueden relacionar dos características dentro de una tabla y el $40 \%$ presentan dificultad para identificar la frecuencia y la moda en una situación estadística. Esto lleva a inferir que los estudiantes se encuentran entre los niveles 1 y 2, razonamiento idiosincrático y razonamiento verbal, primero, por el nivel de formación en que se encuentran los estudiantes (grado tercero) y segundo, por la manifestación evidente de dificultades asociadas al uso del lenguaje estadístico $\mathrm{y}$ aunque entiendan el lenguaje verbal no lo pueden aplicar de manera correcta, por el desconocimiento de los temas estadísticos involucrados.
Este vacío de conocimientos y procedimientos estadísticos y los niveles de razonamiento identificados, pueden tener entre otras las siguientes causas, inferidas al realizar entrevistas a tres docentes de la institución que han impartido clase de matemáticas en diferentes grados de Educación primaria:

Cuando se les indagó sobre los contenidos estadísticos tratados durante el año lectivo y competencias desarrolladas como el razonamiento estadístico, las docentes presentan argumentos como "no alcanza el tiempo" o "se deja para lo último y por diversas actividades no se trabaja", entre otras. Esta situación conlleva a que los niños pierdan la oportunidad de interactuar con la estadística, lo que acarrea, en algunas ocasiones los bajos resultados en las pruebas.

De otra parte, cuando se les preguntó sobre la estrategia para enseñar la competencia razonamiento estadístico a los estudiantes de grado tercero, una profesora responde "los niños aprenden que es una gráfica”. Parece que la enseñanza tiene lugar sin los significados constitutivos de una representación estadística, se cree que saber estadística es solo graficar, memorizar y repetir y es escaso el uso en situaciones del contexto que le sean familiares, significativas y de interés para los estudiantes.

En relación con las dificultades de los alumnos percibidas por los maestros se les formuló la pregunta: ¿Qué dificultades surgen en los niños cuando se les presenta una tabla para 
ser interpretada? Dan respuestas como las siguientes:

\section{"Para el manejo de tablas de barras y estadísticas, si presentan algunas dificultades", "falencias que traen desde preescolar, si hay falencias que se han presentado durante el tiempo de estudio" y "La memo- ria a fallado... ellos no memorizan nada”.}

Esto indica en primer lugar la obsesión por un aprendizaje basado en la memorización, y en segundo lugar, que el razonamiento estadístico conviene desarrollarlo en forma progresiva desde la primaria. En este sentido, las nuevas propuestas curriculares proporcionan una oportunidad para introducir gradualmente los conceptos e ideas estadísticas (Batanero et al., 2013).

Y cuando se les preguntó si ¿¿Ha oído hablar de las fases de la investigación estadística?, dan respuestas como la siguiente:

"Había mejores métodos.... había más exigencia, los niños no eran como actualmente... que a un niño no se le puede llamar la atención fuerte"

Esta respuesta muestra que entre los docentes se desconocen estrategias que facilitan el aprendizaje de la estadística y solo tienen como referencia su proceso de formación para replicar las acciones.

Así, se infiere que los docentes no han desarrollado a cabalidad los contenidos curriculares y estándares de competencias relativos a la estadística en cada grado, y la forma de enseñanza no ha sido muy efectiva, por lo que los niños han sido los menos beneficiados en relación con el aprendizaje de la estadística. Esto, lleva a que ellos no posean los conocimientos estadísticos básicos que para este grado deberían tener, o también es posible que los niños hayan aprendido algunas temáticas de estadística, pero las han olvidado, debido a la metodología tradicional mecánica, memorística, descontextualizada y desprovista de significados.

Por todo lo anterior, es aceptable que la enseñanza de la estadística se realice con base en proyectos, donde los niños experimenten la necesidad de los datos, y ellos mismos sean los que los tomen y recopilen, diligencien las tablas de frecuencias, diseñen las gráficas estadísticas, hagan inferencias con base en las construcciones que ellos han realizado. Caso contrario, una estadística transmisionista y de tablero no sería funcional para un aprendizaje con sentido por parte de los niños.

\section{Conclusiones}

Los resultados de este estudio diagnóstico sobre los niveles iniciales de razonamiento estadístico que manifiestan los estudiantes de grado tercero de la Institución Educativa, refieren dificultades persistentes para comprender y aplicar conceptos y procedimientos relacionados con el pensamiento aleatorio. Muy pocos estudiantes tienen idea de cómo leer e interpretar la información dada en tablas o gráficas estadísticas y leer el contexto desde su cotidianidad; tal vez, 
En el trabajo con proyectos estadísticos se fortalecen aspectos como la comprensión, interpretación y reacción frente a la información estadística, puesto que no solo requiere conocimiento estadístico o matemático, sino también habilidades lingüísticas debido a que los aprendizajes no han partido del entorno o necesidades de los niños.

También, se evidenció que de los conocimientos estadísticos previstos para el grado tercero, según los Estándares Básicos de Competencias y Derechos Básicos de Aprendizaje del Ministerio de Educación Nacional, los niños reflejan en sus protocolos vacíos conceptuales entre los que se encuentran: el conteo, la relación cantidad con extensión, relaciones numéricas, entre otras, y no se puede desconocer que la comprensión lectora también presenta algunas deficiencias por cuanto sería adecuado hacer énfasis en las expresiones lingüísticas más usadas en el contexto de los niños.

En relación con los desempeños de los estudiantes en la competencia de razonamiento, se pudo identificar que casi todas las preguntas eran exigentes en la interpretación y comprensión lectora para los niños de este grado, más cuando el uso de expresiones que no son cotidianas para ellos, lo que hace es confundirlos; de igual manera se puede apreciar la falta de apropiación y aplicación de conceptos y habilidades estadísticas, por parte de los estudiantes, para el desarrollo de procedimientos que les permitan abordar una situación haciendo un análisis adecuado.

El reconocimiento de estas carencias y dificultades en el aprendizaje de la estadística detectadas en el diagnóstico, lleva necesariamente a diseñar una propuesta didáctica que estimule el desarrollo del razonamiento estadístico y promueva el tránsito a niveles de razonamiento superior, junto con la adquisición de una cultura estadística para enfrentar con éxito las situaciones futuras que lo requieran.

En el trabajo con proyectos estadísticos se fortalecen aspectos como la comprensión, interpretación y reacción frente a la información estadística, puesto que no solo requiere conocimiento estadístico o matemático, sino también habilidades lingüísticas, conocimiento del contexto, capacidad para plantear preguntas o problemas y uso adecuado de información para tomar decisiones y buscar soluciones.

Luego, se espera que la estrategia de $\mathrm{ABPr}$ impacte en el desarrollo de habilidades de pensamiento que permita pasar de un conocimiento técnico basado en la fórmulas y la memoria a un conocimiento estratégico es decir pasar de lo conceptual a lo procedimental que es la base fundamental del razonamiento estadístico (Batanero et al., 2013) 


\section{Referencias}

Alvarado, J., \& García, M. (2008). Características más relevantes del paradigma sociocrítico: su aplicación en investigaciones de educación ambiental y de enseñanza de las ciencias realizadas en el Doctorado de Educación del Instituto Pedagógico de Caracas. Sapiens: Revista Universitaria de Investigación, 9(2), 187-202.

Batanero, C. (2013). Sentido estadístico: componentes y desarrollo. En J. M. Contreras, G. R. Cañadas, M. M. Gea y P. Arteaga (Eds.), Actas de las Jornadas Virtuales en Didáctica de la Estadística, Probabilidad y Combinatoria (pp. 55-61). Granada: Departamento de Didáctica de la Matemática de la Universidad de Granada.

Batanero, C., \& Díaz, C. (2004). El papel de los proyectos en la enseñanza y aprendizaje de la estadística. Aspectos didácticos de las matemáticas, 125-164.

Batanero, C., \& Díaz, C. (2011). Estadística con proyectos. Granada: Universidad de Granada.

Batanero, C., Díaz, C., Contreras, J. M., \& Roa, R. (2013). El sentido estadístico y su desarrollo. Números. Revista de Didáctica de las Matemáticas, 83, 7-18.

Casilimas, C. (1996). Investigación cualitativa. Bogotá: Icfes.

Connor, D., Davies, N., \& Payne, B. (2002). Web-based project and key skill work. Teaching

Córdoba, J. (2012). Propuesta para la enseñanza de la estadística en el grado décimo trabajada por proyectos (Doctoral dissertation, Universidad Nacional de Colombia).

Elliot, J. (1994). La investigación acción en educación. Madrid: Morata.

Gil, D., \& Rocha, P. (2010). Contexto escolar y la educación estadística. El proyecto de aula como dispositivo didáctico. Memoria $11^{\circ}$ Encuentro Colombiano de Matemática Educativa.

Godino, J. D., Arteaga, P., Estepa, A., \& Rivas, H. (2013). Desafíos de la enseñanza de la estadística basada en proyectos. En J. M. Contreras, G. R. Cañadas, M. M. Gea y P. Arteaga (Eds.), Actas de las Jornadas Virtuales en Didáctica de la Estadística, Probabilidad y Combinatoria (pp. 173-180). Granada, Departamento de Didáctica de la Matemática de la Universidad de Granada, 2013.

Graham, A. (1987). Statistical investigations in the secondary school. Cambridge: The Open University Centre for Mathematics Education.

Holmes, P. (1997). Assessing project work by external examiners. En I. Gal y J. B. Garfield (Eds.), The assesment challenge in statistics education (pp. 153-164). Voorburg: IOS Press.

Inzunsa, S. (2015). Niveles de interpretación que muestran estudiantes sobre gráficas para comunicar información de contextos económicos y sociodemográficos. Revista 
Mexicana de Investigación Educativa, 20(65), pp.529-555. Recuperado de http://www. redalyc.org/articulo.oa?id=14035408010

Jiménez, A., Limas, L., \& Alarcón, J. (2016). Prácticas pedagógicas matemáticas de profesores de una institución educativa de enseñanza básica y media. Praxis \& Saber, 7(13), 127 - 152. https://doi.org/10.19053/22160159.4169

Ministerio de Educación Nacional (2016). Derechos Básicos de Aprendizaje. Versión 2. Bogotá: Panamericana Formas e Impresos.

NorthWest Regional Educational Laboratory.(s.f.). Aprendizaje por proyectos. Recuperado de http://aprendeenlinea.udea.edu.co/lms/moodle/pluginfile.php/116826/mod_ resource/content/0/tema1/aprendizaje_por_proyectos.pdf

Ríos, J. (2014). Estadística para pequeños estadísticos-Construcción de unidades didácticas y material de apoyo. Tesis de Maestría. Universidad Nacional de Colombia. Manizales.

Tapiero, B., \& Polanco, H. (2014). Desarrollo del razonamiento estadístico a través del trabajo por proyectos. En L. Andrade (Ed.), Memorias del I Encuentro Colombiano de Educación Estocástica (pp. 281-287). Bogotá: Asociación Colombiana de Educación Estocástica. Recuperado de http://funes.uniandes.edu.co/6570/

Tauber, L. (2010). Análisis de elementos básicos de alfabetización estadística en tareas de interpretación de gráficos y tablas descriptivas. Revista Ciencias Económicas, pp. 5374 Recuperado de https://bibliotecavirtual.unl.edu.ar/ojs/index.php/CE/article/ download/1146/1782

Zapata, L. (2011). ¿Cómo contribuir a la alfabetización estadística? Revista Virtual Universidad Católica del Norte, 1(33), 234-247. Recuperado de http://revistavirtual. ucn.edu.co/index.php/RevistaUCN/article/viewFile/4/8 